\title{
Prediction Variance Properties of Third-Order Response Surface Designs in the Hypersphere
}

\author{
Abimibola Victoria Oladugba, Brenda Mbouamba Yankam* \\ Department of Statistics, University of Nigeria, Nsukka, Nigeria
}

Received October 8, 2021; Revised November 26, 2021; Accepted December 13, 2021

\section{Cite This Paper in the following Citation Styles}

(a): [1] Abimibola Victoria Oladugba, Brenda Mbouamba Yankam, "Prediction Variance Properties of Third-Order Response Surface Designs in the Hypersphere," Mathematics and Statistics, Vol. 10, No. 1, pp. 61 - 73, 2022. DOI: 10.13189/ms.2022.100105.

(b): Abimibola Victoria Oladugba, Brenda Mbouamba Yankam (2022). Prediction Variance Properties of Third-Order Response Surface Designs in the Hypersphere. Mathematics and Statistics, 10(1), 61 - 73. DOI: 10.13189/ms.2022.100105.

Copyright $\bigcirc 2022$ by authors, all rights reserved. Authors agree that this article remains permanently open access under the terms of the Creative Commons Attribution License 4.0 International License

\begin{abstract}
The variance dispersion graphs (VDGs) and the fraction of design space (FDS) graphs are two graphical methods that effectively describe and evaluate the points of best and worst prediction capability of a design using the scaled prediction variance properties. These graphs are often utilized as an alternative to the single-value criteria such as $D$ - and $E$ - when they fail to describe the true nature of designs. In this paper, the VDGs and FDS graphs of third-order orthogonal uniform composite designs $\left(\mathrm{OUCD}_{4}\right)$ and orthogonal array composite designs $\left(\mathrm{OACD}_{4}\right)$ using the scaled-prediction variance properties in the spherical region for 2 to 7 factors are studied throughout the design region and over a fraction of space. Single-valued criteria such as $D$-, $A$ - and $G$-optimality are also studied. The results obtained show that the $\mathrm{OUCD}_{4}$ is more optimal than the $\mathrm{OACD}_{4}$ in terms of $D$-, $A$ - and $G$-optimality. The $\mathrm{OUCD}_{4}$ was shown to possess a more stable and uniform scaled-prediction variance throughout the design region and over a fraction of design space than the $\mathrm{OACD}_{4}$ although the stability of both designs slightly deteriorated towards the extremes.
\end{abstract}

Keywords Scaled-Prediction Variance, Variance Dispersion Graphs, Fraction of Design Space Graphs Orthogonal Uniform Composite Designs, Spherical Region, $G$-Optimality Criterion

\section{Introduction}

Third-order response surface designs are flexible designs that estimate the linear, bilinear (interaction), quadratic and cubic terms by estimating all the parameters of the third-order model [1-4]. These third-order response surface designs provide more accurate responses to the prediction of more complex systems [5]. They are successfully used to establish a relationship between the independent variables and the responses when the model is unjustifiably linear, and are robust under certain conditions, provide an estimate of the pure experimental error and perform lack of fit test especially when the second-order response surface designs fail [3, 5-7]. Although the second-order response surface designs are the simplest to use because they are cost-effective, they are not always the most efficient when a lack of fit is detected due to the presence of higher-order terms. Third-order terms must be added to the second-order model to achieve a better approximation of the actual response function under consideration, resulting in the third-order model that is fitted onto the third-order response surface design. The third-order response surface designs consist mainly of three portions; the factorial portions having two-levels, the axial portion having at least four-levels and some center points [2-4].

Many third-order response surface designs have been studied by several authors. For example, Arshad et al. [3] constructed third-order augmented Box-Behnken designs to estimate the third-order model. Rashid et al. [2] constructed third-order augmented fractional 
Box-Behnken designs. Kwon and Park [8] studied extended central composite designs (ECCDs) and the conditions for slope-rotatability to estimate the third-order model. Yang [5] studied third-order response surface designs by applying multiple optimality criteria. Zhang et al. [4] constructed third-order orthogonal array composite designs $\left(\mathrm{OACD}_{4}\right)$ to estimate the third-order model parameters. Yankam and Oladugba [9] constructed third-order orthogonal uniform composite designs $\left(\mathrm{OUCD}_{4}\right)$ to estimate the third-order model parameters.

These designs are commonly selected using the best single-valued criteria, such as D-optimality, which considers the variance and covariances of the model's coefficient estimates, I-optimality, which considers the average variance for a projected value over the region of interest, and G-optimality, which considers the overall variance for a projected value over the region of interest [10-14]. Unfortunately, single-valued criteria frequently fail to reflect the true nature of a design employed to fit the response surface model in terms of prediction properties over a specified region of interest or portion of the design space $[12,15]$. When comparing response surface designs using optimality criteria such as $D-, I-$, and $G$-, it is crucial to remember that a design may perform well under one optimality criterion but not under another. Choosing an optimality criterion may therefore be required before selecting a design. Hence, when a design is reduced to a single-valued criterion, some information may be lost with regard to the quality of the prediction variance [16]. Thus, the prediction variance is an important property that effectively describes the stability and uniformity of a design throughout the design region or over a fraction of the design space, showing effectively and efficiently the region of best and worst prediction presented in form of graphs called the variance dispersion graphs (VDGs) and the fraction of design space (FDS) graphs [17-18].

The VDG and FDS graphs were introduced as a substitute for the single-valued criterion for evaluating response surface designs' prediction capability. The VDG introduced by Giovannitti-Jensen and Myers [16], is used to evaluate the stability and uniformity of the prediction variance properties of designs in the region of interest or where the region has best and or worst prediction variance, thus, helping the researcher to have better knowledge of points of best and worst predictions. The FDS graph was first introduced by Zahran et al. [17] to complement the VDG. These graphs have been widely utilized to study the behaviour of the prediction capabilities of response surface designs over the entire design region and over a fraction of the design region [11, 13-14, 17, 19-21]. Many authors have studied the prediction variance properties of some second-order designs. Take for instance Borkowski [15] studied the scaled prediction variance of central composite designs (CCDs) and Box-Benhken designs (BBDs); Wesley et al. [22] studied the prediction variance properties of split-plot designs in both the spherical and cuboidal region; Borkowski [12] studied the prediction variance capabilities of CCDs and BBDs in the spherical region; Chigbu et al. [23] studied the prediction variance properties of some composite designs in the spherical region; Onwuamaeze [24] investigated the prediction variance properties of several cuboidal designs.; Zahran et al. [17] used the FDS graphs to assess the prediction capability of some designs; Oyejola and Nwanya [13] studied the prediction capability of inscribed central composite designs, by using FDS graphs; Nwanya and Dozie [14] used FDS graph to assess the prediction capability of some designs. The VDG is obtained by plotting scaled prediction variances against the radius $r$, of the region of interest while the FDS graph is obtained by plotting the scaled prediction variances against a fraction of space [13, 18]. There is, however, little or no information on the prediction variance properties of third-order response surface designs in the literature.

In this paper, the prediction variance properties of two third-order designs; orthogonal uniform composite designs $\left(\mathrm{OUCD}_{4}\right)$ introduced by Yankam and Oladugba [9] and orthogonal array composite designs $\left(\mathrm{OACD}_{4}\right)$ introduced by Zhang et al. [4] using VDG and FDS graphs are studied. These designs have several advantages, including the ability to use resolution IV design for factor screening, the capacity to execute in-depth analysis, the ability to provide more flexible run sizes than other types of composite designs, and the ability to perform multiple analyses for cross-validation. More specifically, the $\mathrm{OUCD}_{4}$ are good space-filling designs whose design points are evenly distributed in the experimental region as a result of the uniform design in the $\mathrm{OUCD}_{4}$. The motivation of using these two third-order response surface designs is to demonstrate how flexible these designs are and project their prediction variance properties in the region of interest. These designs are also evaluated in terms of single-valued criteria such as $D$-, $A$ - and $G$-optimality criteria to show the design performance.

The remainder of this paper is divided into 5 Sections, which are as follows: Section 2 gives a brief introduction to third-order response surface designs and also provides the model description for these designs. In Section 3, the single-value criteria used in this study are discussed. Section 4 describes the VDG and FDS graphs. Section 5 provides a comparison of the $\mathrm{OUCD}_{4}$ and $\mathrm{OACD}_{4}$ in terms of $D-, A$ - and $G$-optimality criteria, VDG and FDS graphs. The conclusion of this study is provided in Section 6.

\section{Third-Order Response Surface Designs}

This section describes the construction and composition of orthogonal uniform composite designs $\left(\mathrm{OUCD}_{4}\right)$ and orthogonal array composite designs $\left(\mathrm{OACD}_{4}\right)$ and also 
provides the model description of these third-order response surface designs.

\subsection{Orthogonal Uniform Composite Designs $\left(\mathrm{OUCD}_{4}\right)$}

The orthogonal uniform composite design $\left(\mathrm{OUCD}_{4}\right)$ is a new third-order response surface design introduced by Yankam and Oladugba [9]. These $\mathrm{OUCD}_{4}$ are good space-filling designs that are nearly rotatable and nearly orthogonal third-order response surface designs. They are composed of three parts namely: (i) a factorial portion with factor levels coded as $[-1,1]$ and the number of factorial points is denoted by $n_{f}$; (ii) a four-level uniform design (UD) as the axial portion with all $x_{i}=-\alpha,-\frac{\alpha}{\alpha}$, $\frac{\alpha}{a}$, or $\alpha(\alpha>0 ; a=2,3,4, \ldots)$ and the number of axial points is denoted by $n_{\alpha}$, and (iii) center points with all $x_{i}=0$ and the number of center points is denoted by $n_{\alpha}$, giving a total of $N=n_{f}+n_{\alpha}+n_{0}$.

To construct an $\mathrm{OUCD}_{4}$, a two-level factorial design and a four-level uniform design (UD) are selected. The two-level factorial design is obtained by choosing either a $2^{k}$ full factorial if $k \leq 4$ or a regular $2^{k-l}$ fractional factorial design that is minimum aberration i.e., a design with maximum resolution. With $k$ being the number of factors and $l$ the design generator. The two-level factorial design was constructed by using Minitab statistical software version 16 .

As regarding the four-level uniform designs (UDs), let $U D\left(N, S_{1}, \ldots, S_{k}\right)$ be a $k$ factor UD-type designs in which each of the $S_{j}$ levels i.e., $\left(1, \ldots, S_{j}\right)$, occur $N / S_{j}$ times in the $j^{\text {th }}$ column, $(j=1, \ldots, k)$, denoted by $U D\left(N, S^{k}\right)$ when $S_{1}=\cdots=S_{k}=S$, with UD being the uniform design, $N$ the number of design points, $S$ the number of factor's levels and $k$ the number of factors. A uniform UD-type design is chosen under the mixture discrepancy criterion (MD) by using the arguments init= "rand", crit= "MD", and maxiter $=100$ from the $\mathrm{R}$ package named UniDOE of Zhang et al. [25] (http://rmirror.lau.edu.lb/web/packages/UniDOE/UniDOE .pdf). The axial portion i.e., $\alpha$ is chosen at the spherical region such that $\alpha=\sqrt{k}$. This, therefore, give the spherical $\mathrm{OUCD}_{4}$ in which all axial design points are located on a sphere. Consider the example presented in Table 1 for a 29 -run $\mathrm{OUCD}_{4}$ for factor 3 . This is a simple combination of a minimum aberration $2^{3}$ design with a mixture discrepancy three-column uniform design, $U D\left(16,4^{3}\right)$ for factor 3 with 5 center points.

Table 1. A general structure of 29-runs $\mathrm{OUCD}_{4}$ for factor 3 with $n_{0}=5$ center point

\begin{tabular}{|c|c|c|c|c|}
\hline & \multirow{3}{*}{ Runs } & \multicolumn{3}{|c|}{ Factors } \\
\hline & & \multicolumn{3}{|c|}{3 factors $\mathrm{OUCD}_{4}$ with 29-runs } \\
\hline & & $\mathbf{A}$ & B & C \\
\hline Factorial portion $\left(\boldsymbol{n}_{\boldsymbol{f}}\right)$ & $\begin{array}{l}1 \\
2 \\
3 \\
4 \\
5 \\
6 \\
7 \\
8\end{array}$ & $\begin{array}{c}-1 \\
1 \\
1 \\
-1 \\
1 \\
-1 \\
-1 \\
1\end{array}$ & $\begin{array}{c}1 \\
-1 \\
-1 \\
-1 \\
1 \\
-1 \\
1 \\
1\end{array}$ & $\begin{array}{c}1 \\
1 \\
-1 \\
-1 \\
1 \\
1 \\
-1 \\
-1\end{array}$ \\
\hline Axial portion $\left(\boldsymbol{n}_{\alpha}\right)$ & $\begin{array}{c}9 \\
10 \\
11 \\
12 \\
13 \\
14 \\
15 \\
16 \\
17 \\
18 \\
19 \\
20 \\
21 \\
22 \\
23 \\
24 \\
\end{array}$ & $\begin{array}{c}1 \\
1 \\
1 \\
1 / 3 \\
1 / 3 \\
-1 / 3 \\
-1 \\
1 / 3 \\
-1 \\
-1 / 3 \\
-1 \\
-1 / 3 \\
1 / 3 \\
-1 / 3 \\
-1 \\
1 \\
\end{array}$ & $\begin{array}{c}-1 \\
-1 / 3 \\
1 / 3 \\
-1 / 3 \\
-1 \\
1 / 3 \\
1 \\
-1 / 3 \\
1 \\
-1 / 3 \\
-1 \\
1 / 3 \\
-1 \\
1 \\
1 / 3 \\
1 \\
\end{array}$ & $\begin{array}{c}1 \\
-1 \\
-1 / 3 \\
-1 \\
1 / 3 \\
-1 / 3 \\
1 \\
1 / 3 \\
1 \\
1 \\
-1 / 3 \\
-1 / 3 \\
1 / 3 \\
-1 \\
-1 \\
1 / 3\end{array}$ \\
\hline Center runs replicate $\left(\boldsymbol{n}_{\mathbf{0}}\right)$ & $\begin{array}{l}25 \\
26 \\
27 \\
28 \\
29\end{array}$ & $\begin{array}{l}0 \\
0 \\
0 \\
0 \\
0\end{array}$ & $\begin{array}{l}0 \\
0 \\
0 \\
0 \\
0\end{array}$ & $\begin{array}{l}0 \\
0 \\
0 \\
0 \\
0\end{array}$ \\
\hline
\end{tabular}




\subsection{Orthogonal Array Composite Designs $\left(\mathrm{OACD}_{4}\right)$}

The $\mathrm{OACD}_{4}$ is a new class of third-order designs introduced by Zhang et al. [4] and it is composed of three parts namely: (i) two-level orthogonal array (OA) as the factorial portion with factor levels given by $[-1,1]$ denoted by $d_{1}$; (ii) four-level orthogonal array (OA) as the axial portion with levels given by $x_{i}=-\alpha,-\frac{\alpha}{a}, \frac{\alpha}{a}$, or $\alpha(\alpha>$ $0 ; a=2,3,4, \ldots$ ) denoted by $d_{2}$, and (ii) center points with $x_{i}=0$ denoted $d_{0}$. Hence, the $\mathrm{OACD}_{4}$ uses a total of $N=d_{1}+d_{2}+d_{0}$. The four-level portion $d_{2}$ of $\mathrm{OACD}_{4}$ was constructed by taking the first $k$ columns of "oa.16.5.4.2.txt" and "oa.32.9.4.2.txt" from Sloane's website http://neilsloane.com/oadir/.

\subsection{Model Description}

For $k$ independent variables, the fitted third-order model used in this study is defined in equation (1) as;

$$
\begin{aligned}
y=\beta_{0}+\sum_{i=1}^{k} \beta_{i} x_{i} & +\sum_{i=1}^{k} \beta_{i i} x_{i}^{2}+\sum_{i=1}^{k-1} \sum_{j=i+1}^{k} \beta_{i j} x_{i} x_{j}+ \\
& +\sum_{i=1}^{k} \beta_{i i i} x_{i}^{3}+\varepsilon
\end{aligned}
$$

where $y$ represents the response, $\beta_{0}, \beta_{i}, \beta_{i i}, \beta_{i j}$ and $\beta_{i i i}$ denote the intercept, linear coefficient, quadratic coefficient, bilinear (interaction) coefficient and cubic coefficient, respectively, and $\varepsilon$ denotes the random experimental error with $\varepsilon \sim N\left(0\left(\sigma^{2}\right)\right.$.

The matrix for of the third-order model is defined as:

$$
f^{\prime}(x)=\left[f_{1}^{\prime}(x), f_{2}^{\prime}(x), f_{3}^{\prime}(x), f_{4}^{\prime}(x)\right]
$$

where

$$
\begin{gathered}
f_{1}^{\prime}(x)=\left(1, x_{1}, \ldots, x_{k}\right) \\
f_{2}^{\prime}(x)=\left(x_{1}^{2}, \ldots, x_{k}^{2}\right) \\
f_{3}^{\prime}(x)=x_{1} x_{2}, \ldots, x_{k-1} x_{k} \\
f_{4}^{\prime}(x)=x_{1}^{3}, \ldots, x_{k}^{3}
\end{gathered}
$$

\section{Single-Value Criteria}

The $D$-, $A$ - and $G$-optimality criteria used to evaluate the $\mathrm{OUCD}_{4}$ and $\mathrm{OACD}_{4}$ are described in this section.

\subsection{D-optimality Criterion}

The $D$-efficiency is a criterion that maximizes the determinant of the information matrix of a design and it is defined in equation (3) as;

$$
D_{e f f}(d)=\frac{1}{n}\left(\left|X^{\prime} X\right|\right)^{1 / p}
$$

where $X^{\prime} X$ is the information matrix and $p$ the number parameters of the model under study.

\subsection{A-optimality Criterion}

(4) as; $A$-efficiency is a criterion that minimizes the sum of variances of the model coefficients which is given in equation (4) as;

$$
A_{\text {opt }}(d)=\left(\frac{1}{p} \operatorname{trace}^{-1}\right)^{-1}
$$

where $M=X^{\prime} X$ is the normalized moment matrix and $p$ is the parameter of the third-order model.

\subsection{G-optimality Criterion}

The G-optimality criterion minimizes a design's maximum prediction variance and it's defined in equation (5) as;

$$
G_{\text {opt }}(d)=\frac{p}{v(\underline{x})_{\max }}
$$

where $v(\underline{x})_{\text {max }}=\max \left(n \cdot \operatorname{var} \frac{[\hat{y}(x)]}{\sigma^{2}}\right)=\left(n \cdot x^{\left.\prime\left(X^{\prime} X\right)^{-1} x\right)}\right.$ is the scale prediction variance, with $x=\left(1, x_{1}, x_{2}, \ldots, x_{k} ; x_{1}^{2}, x_{2}^{2}, \ldots, x_{k}^{2} ; x_{1} x_{2}, \ldots, x_{k-1} x_{k} ; x_{1}^{3}\right.$, $\left.x_{2}^{3}, \ldots, x_{k}^{3}\right)$ vector of design points.

\section{Graphical Assessment Techniques}

This section describes the VDG and FDS graphs used to evaluate the third-order designs prediction variance.

\subsection{Variance Dispersion Graph}

Giovannitti-Jensen and Myers [16] developed the VDG to examine the consistency and uniformity of response surface designs' prediction variance properties. The VDG is comprised of three properties, the maximum, average and minimum scaled variance of a predicted value on a hypersphere given a spherical radius. The variance of a predicted value at a given point $x_{1}, \ldots, x_{k}$ in the experimental region is defined in equation (6) as:

$$
\operatorname{var}[\hat{y}(x)]=\sigma^{2} x^{\prime}\left(X^{\prime} X\right)^{-1} x
$$

where $x=\left(1, x_{1}, x_{2}, \ldots, x_{k} ; x_{1}^{2}, x_{2}^{2}, \ldots, x_{k}^{2} ; x_{1} x_{2}, \ldots\right.$, $\left.x_{k-1} x_{k} ; x_{1}^{3}, x_{2}^{3}, \ldots, x_{k}^{3}\right)$ and $X$ is an $n \times p$ model matrix derived from the $n \times k$ design matrix.

The maximum scale prediction variance (SPV) used to compare the designs in this study is defined in equation (7) as:

$$
\begin{gathered}
\max \frac{n \operatorname{vvar}[\hat{y}(x)]}{\sigma^{2}}=\max \left(n \cdot x^{\prime}\left(X^{\prime} X\right)^{-1} x\right)= \\
=1+n\left(\lambda_{\max }\right) r^{2}
\end{gathered}
$$

The minimum SPV in this study is defined in equation (8) as:

$$
\begin{gathered}
\min \frac{n \operatorname{var}[\hat{y}(x)]}{\sigma^{2}}=\min \left(n \cdot x^{\prime}\left(X^{\prime} X\right)^{-1} x\right)= \\
=1+n\left(\lambda_{\min }\right) r^{2}
\end{gathered}
$$

The average SPV in this study is defined in equation (9) as:

$$
V^{r}=1+\frac{n r^{2}}{k} \sum_{i=1}^{k} \lambda_{i}
$$


where $\lambda_{\max }, \lambda_{\min }$ and $\lambda_{i}$ are the maximum, minimum and $i^{\text {th }}$ eigenvalues of $\left(X^{\prime} X\right)^{-1}$ respectively. A stable and uniform SPV is often desired and it is associated with smooth and flat VDG curves [18]. The VDGs of OUCD compared with $\mathrm{OACD}_{4}$ when the region is spherical are presented in Figure 1-6 for $2 \leq k \leq 7$.

\subsection{Fraction of Design Space Graph}

The FDS graph was first introduced by Zahran et al. [17] to complement the variance dispersion graph. The FDS graph depicts the SPV's characteristics across a multidimensional region on a two-dimensional graph with a single curve. The FDS graph shows the fraction of the design space at any SPV value. It is obtained by randomly selecting a large number of variables, say $u$. The FDS is defined in equation (10) as;

$$
\begin{array}{r}
F D S=\frac{1}{\Psi} \int, \ldots, \int I(X) d x_{1}, \ldots, d x_{k} \\
\text { where } I(X)=\left\{\begin{array}{cc}
0.5 \frac{(|h|-h)}{h} ; & h \neq 0 \\
0 ; & h=0
\end{array}\right.
\end{array}
$$

$h=u(x)-u, u$ is the scaled-prediction variance value, $u(x)$ is the prediction variance such that $u(x)<u, h<0$ and $\Psi=2^{k}$ is the volume of the design space. The $\mathrm{x}$-axis shows the design space quantiles, which range from 0 to 1 , while the $\mathrm{y}$-axis displays the SPV values. According to Zahran et al. [17], a larger fraction of design space is often desired.

\section{Comparison of the Designs}

This section compares the $D$-, $A$ - and $G$-optimality criteria, VDG and FDS graph of $\mathrm{OUCD}_{4}$ and $\mathrm{OACD}_{4}$

\subsection{Comparison Using $D$-, $A$ - and $G$-optimality Criteria}

In comparing response surface designs, a design with the smallest optimality criterion value is often desired over those that are not [13-15]. The results of the $D$-, $A$ - and $G$-optimality criteria obtained when the radius of a sphere is $\alpha=\sqrt{k}$ are presented in Table 2. Table 2 shows that the $\mathrm{OUCD}_{4}$ fared better than the $\mathrm{OACD}_{4}$ for $2 \leq k \leq 7$, with lesser $G$ - and $A$-optimality values. The only exception where the $\mathrm{OUCD}_{4}$ did not perform well is when $k=6$. The good performance of the $\mathrm{OUCD}_{4}$ could be as a result of the uniform design in $\mathrm{OUCD}_{4}$ which causes the design to be more flexible over the $\mathrm{OACD}_{4}$. Comparison of the $\mathrm{OUCD}_{4}$ and $\mathrm{OACD}_{4}$ in terms of $D$-optimality shows that the $\mathrm{OUCD}_{4}$ performed better for $3 \leq k \leq 7$ compared to the $\mathrm{OACD}_{4}$, the only exception where the $\mathrm{OACD}_{4}$ performed better is when $k=2$. In general, the $\mathrm{OUCD}_{4}$ and $\mathrm{OACD}_{4}$ are good optimal third-order response surface designs, although the $\mathrm{OUCD}_{4}$ performed better than the $\mathrm{OACD}_{4}$.

\subsection{Comparison Using VDG}

The comparison in subsection 5.1 was done by using the singled-value criteria that do not describe how well a design performed in the design region which was estimated. As a result, the VDGs which show how well a design performed in the design region, are obtained and presented in Figures 1 to 6.

\begin{tabular}{|c|c|c|c|c|c|c|c|c|}
\hline Design & $\boldsymbol{k}$ & $p$ & $\alpha$ & $n_{f}$ & $N$ & $D$-opt & $A$-opt & $G$-opt \\
\hline $\mathrm{OUCD}_{4}$ & 2 & 8 & 1.4142 & 4 & 25 & 0.6093 & 0.1936 & 0.2353 \\
\hline $\mathrm{OACD}_{4}$ & 2 & 8 & 1.4142 & 4 & 25 & 0.5604 & 0.2268 & 0.3333 \\
\hline $\mathrm{OUCD}_{4}$ & 3 & 13 & 1.7321 & 8 & 29 & 1.0644 & 0.3602 & 0.0879 \\
\hline $\mathrm{OACD}_{4}$ & 3 & 13 & 1.7321 & 8 & 29 & 1.1558 & 0.4754 & 0.1764 \\
\hline $\mathrm{OUCD}_{4}$ & 4 & 19 & 2.0000 & 16 & 37 & 1.5392 & 0.5514 & 0.0330 \\
\hline $\mathrm{OACD}_{4}$ & 4 & 19 & 2.0000 & 16 & 37 & 1.6378 & 0.6480 & 0.0544 \\
\hline $\mathrm{OUCD}_{4}$ & 5 & 26 & 2.2361 & 16 & 37 & 1.7133 & 0.4921 & 0.0196 \\
\hline $\mathrm{OACD}_{4}$ & 5 & 26 & 2.2361 & 16 & 37 & 1.8544 & 0.6076 & 0.0305 \\
\hline $\mathrm{OUCD}_{4}$ & 6 & 34 & 2.4495 & 32 & 96 & 2.9317 & 0.8821 & 0.0283 \\
\hline $\mathrm{OACD}_{4}$ & 6 & 34 & 2.4495 & 32 & 96 & 3.3484 & 1.0335 & 0.0168 \\
\hline $\mathrm{OUCD}_{4}$ & 7 & 43 & 2.6458 & 64 & 101 & 2.9528 & 1.0071 & 0.0144 \\
\hline $\mathrm{OACD}_{4}$ & 7 & 43 & 2.6458 & 64 & 101 & 3.1977 & 1.0738 & 0.0132 \\
\hline
\end{tabular}

Table 2. Designs examined under the $D$-, $A$ - and $G$-optimality criteria in the spherical region for $\alpha=\sqrt{k}$ and $n_{0}=5$ center points 
From Figures 1 to 6 , for $2 \leq k \leq 7$, we observed that the $\mathrm{OUCD}_{4}$ and $\mathrm{OACD}_{4}$ have a stable and uniform SPV throughout the design region for the minimum and average SPV meanwhile for the maximum SPV, we observe a slight deterioration towards the extreme. We also observed that the maximum SPV of the $\mathrm{OUCD}_{4}$ deteriorates slightly more than the maximum SPV of $\mathrm{OACD}_{4}$ as observed in Figures 1 to 4 , the average SPV of $\mathrm{OACD}_{4}$ deteriorates more than the average SPV of $\mathrm{OUCD}_{4}$ as shown in Figures 1 to 3 and the minimum SPV of $\mathrm{OUCD}_{4}$ and $\mathrm{OACD}_{4}$ coincide as shown in Figures 3 and 6 although the minimum SPV of $\mathrm{OUCD}_{4}$ is better in most cases than those of $\mathrm{OACD}_{4}$ as shown in Figures 1, 2, 4 and 6 with a very stable and uniform SPV throughout the design region. The presence of the uniform design in the $\mathrm{OUCD}_{4}$ contributed greatly to the stability and uniformity of the $\mathrm{OUCD}_{4}$ over $\mathrm{OACD}_{4}$ although it is not easy to choose the best design among these two designs based on their VDGs. We observed a clear picture of the SPV of $\mathrm{OUCD}_{4}$ and $\mathrm{OACD}_{4}$ over the entire design region compared to when the single-value criteria are used where we cannot for sure say if the result is projected over the entire design region. Thus, the VDG is a good way of observing designs prediction variance properties than single-value criteria.

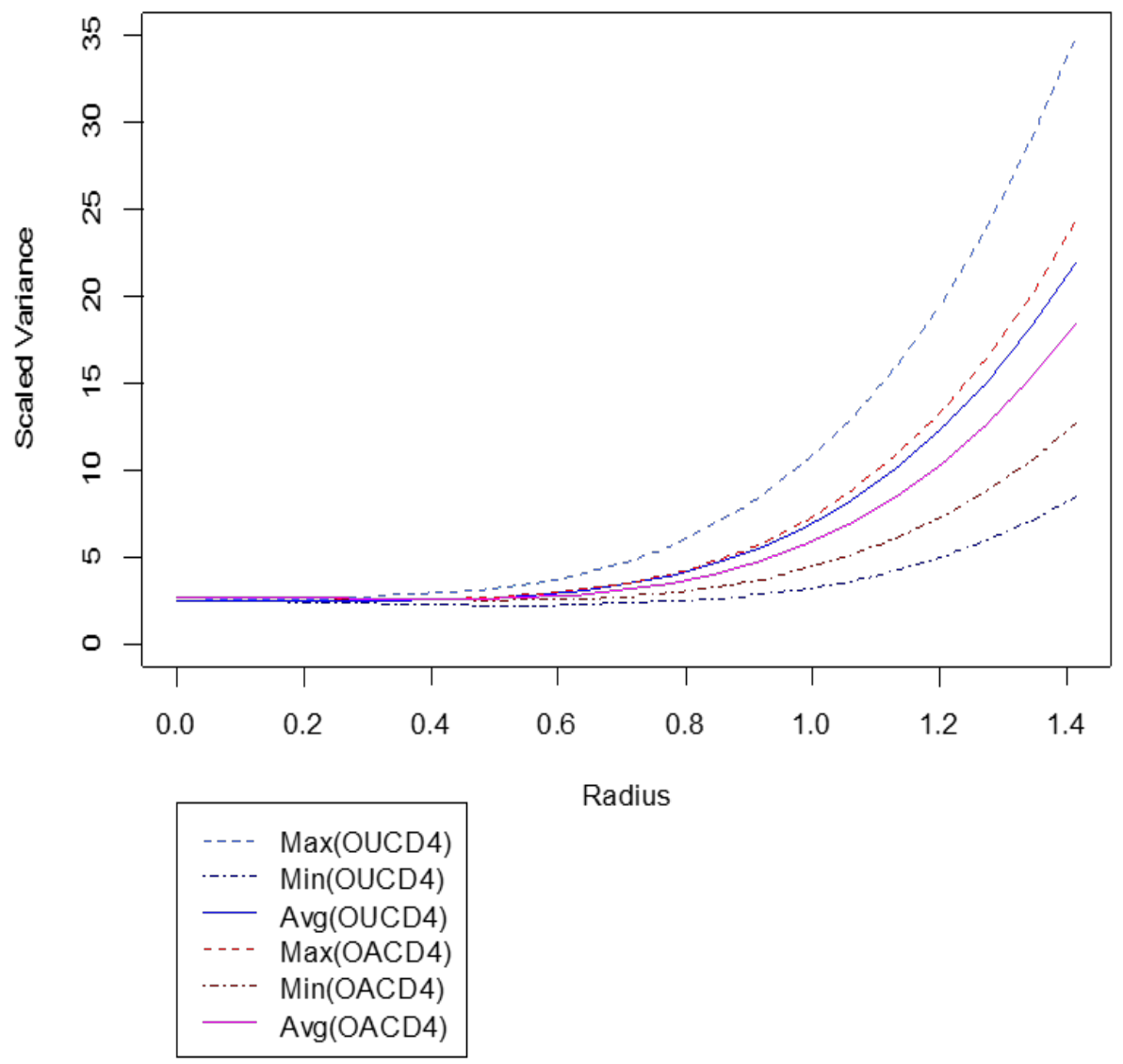

Figure 1. VDG for $\mathrm{OUCD}_{4}$ and $\mathrm{OACD}_{4}$ when $k$ is 2 


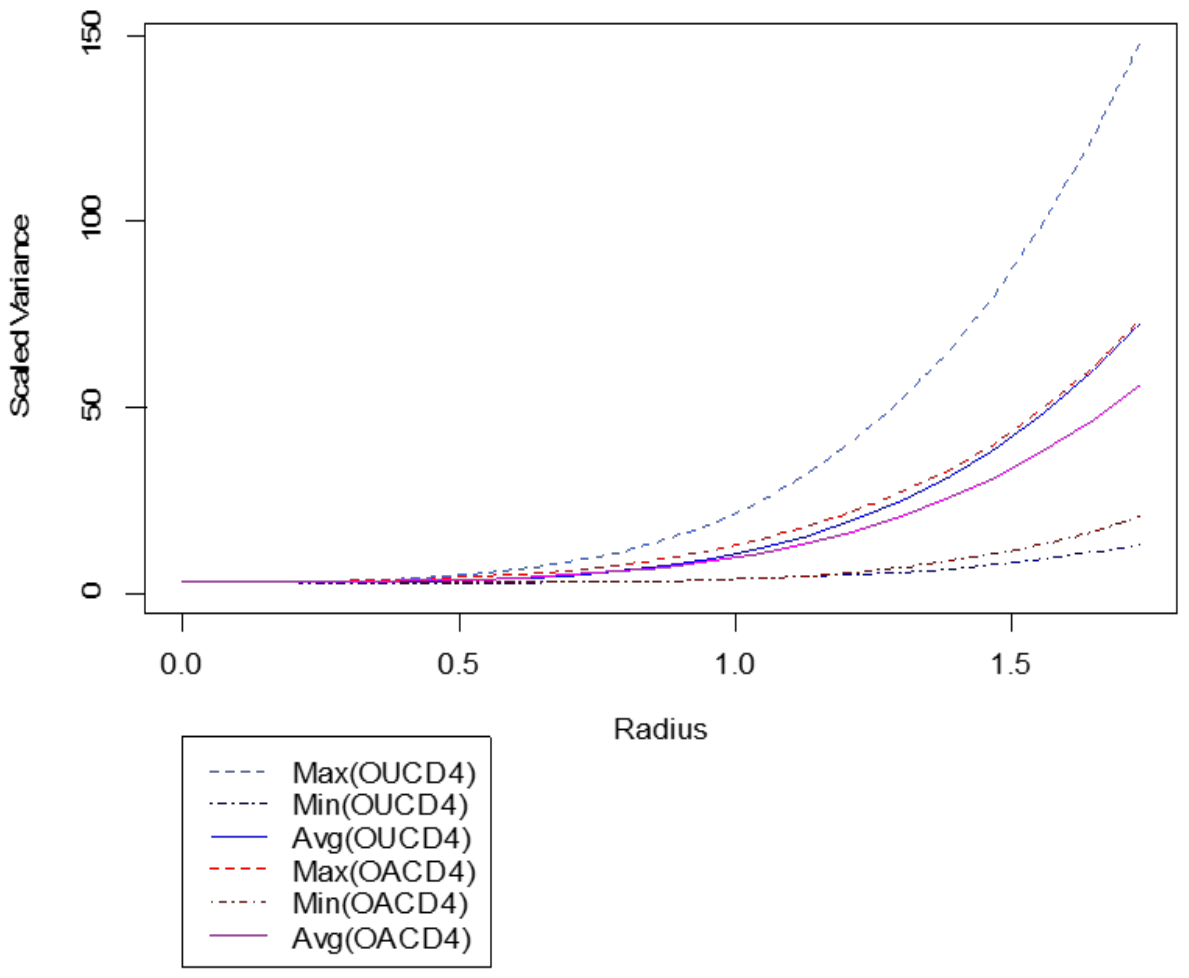

Figure 2. VDG for $\mathrm{OUCD}_{4}$ and $\mathrm{OACD}_{4}$ when $k$ is 3

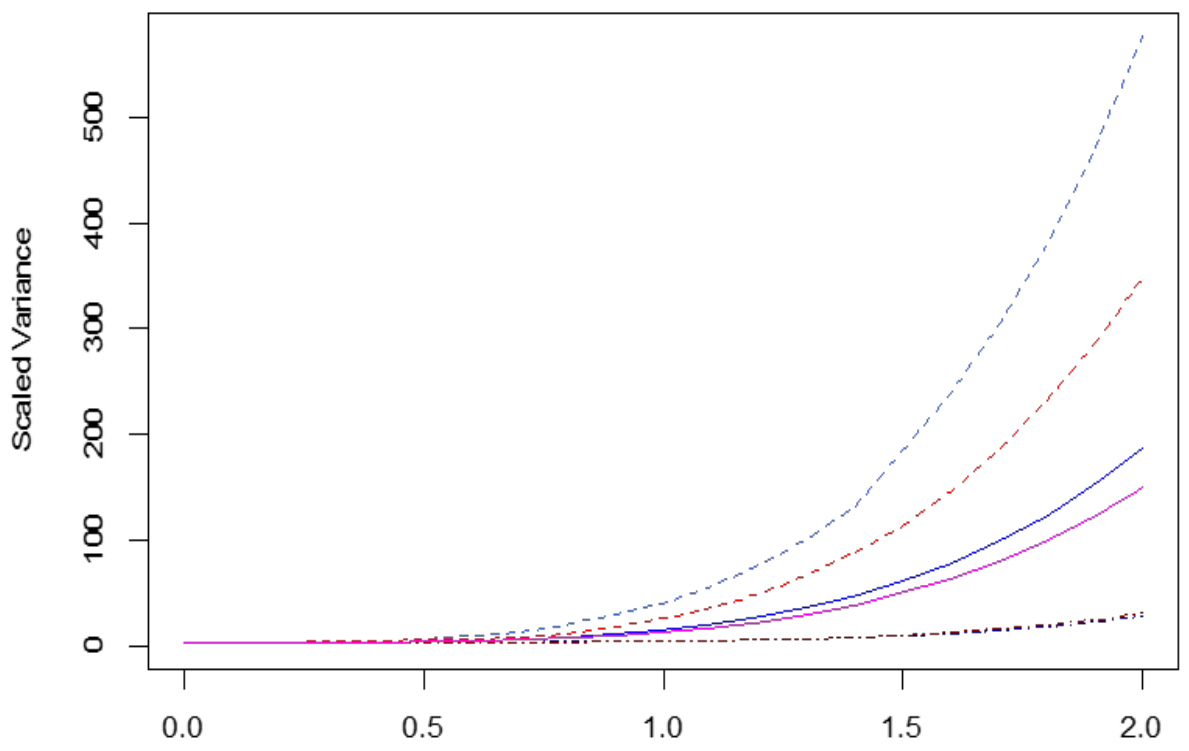

Radius

$$
\begin{array}{ll}
-\cdots & \text { Max(OUCD4) } \\
\cdots-\cdots & \text { Min(OUCD4) } \\
-\cdots & \text { Avg(OUCD4) } \\
-\cdots & \text { Max(OACD4) } \\
\cdots & \text { Min(OACD4) } \\
- & \text { Avg(OACD4) }
\end{array}
$$

Figure 3. VDG for $\mathrm{OUCD}_{4}$ and $\mathrm{OACD}_{4}$ when $k$ is 4 


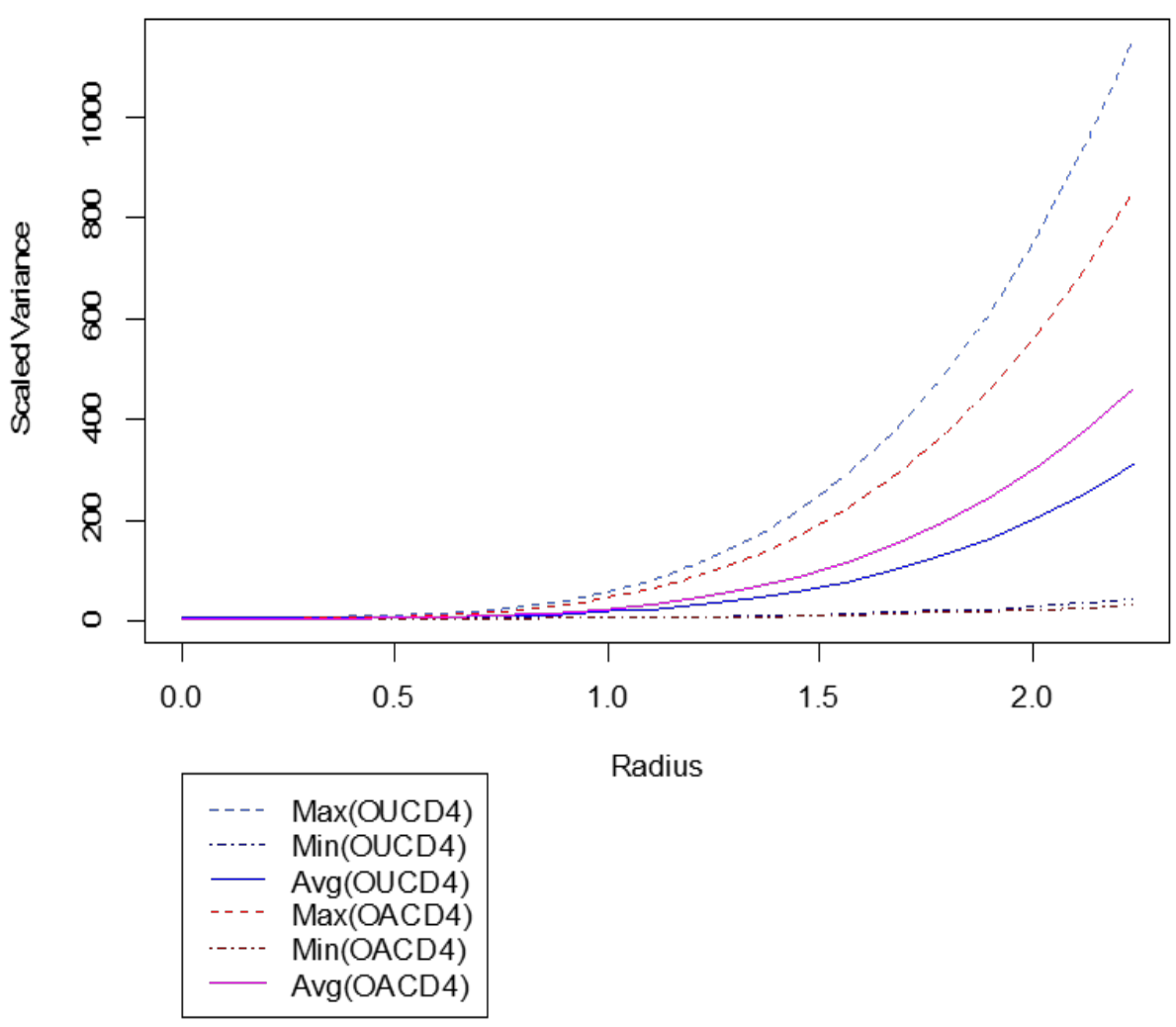

Figure 4. VDG for $\mathrm{OUCD}_{4}$ and $\mathrm{OACD}_{4}$ when $k$ is 5

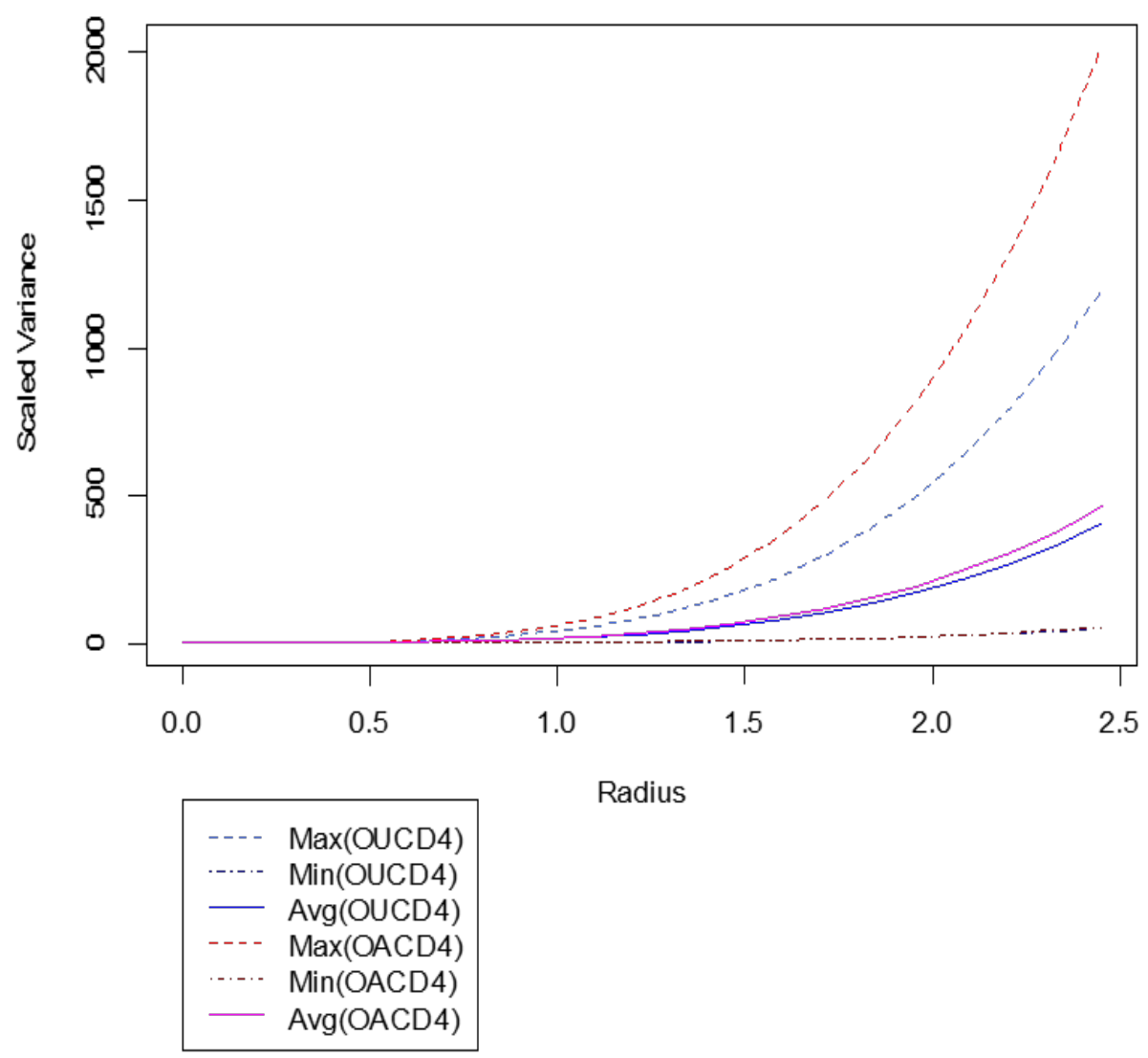

Figure 5. VDG for $\mathrm{OUCD}_{4}$ and $\mathrm{OACD}_{4}$ when $k$ is 6 


\subsection{Comparison Using the FDS Graphs}

The FDS graphs used to complement the VDG were obtained by using equation (9) and the results are presented in Figures 7 to 12.

From Figures 7 to 13 for $2 \leq k \leq 7$, it was observed that when the factors are $2,3,4$, and 6 the $\mathrm{OUCD}_{4}$ performed better with maximum SPV compared to the
$\mathrm{OACD}_{4}$ throughout a fraction of design space. This however does not apply to factors 5 and 7 since, in factors 5 and 7, the SPV of $\mathrm{OUCD}_{4}$ and $\mathrm{OUCD}_{4}$ coincide, thus performing almost the same. The good performance of the $\mathrm{OUCD}_{4}$ over the $\mathrm{OACD}_{4}$ is explained by the presence of the uniform design in the $\mathrm{OUCD}_{4}$ which causes it to have a better SPV observed in the FSD graphs. Thus, causing the $\mathrm{OUCD}_{4}$ to perform relatively better than the $\mathrm{OACD}_{4}$.

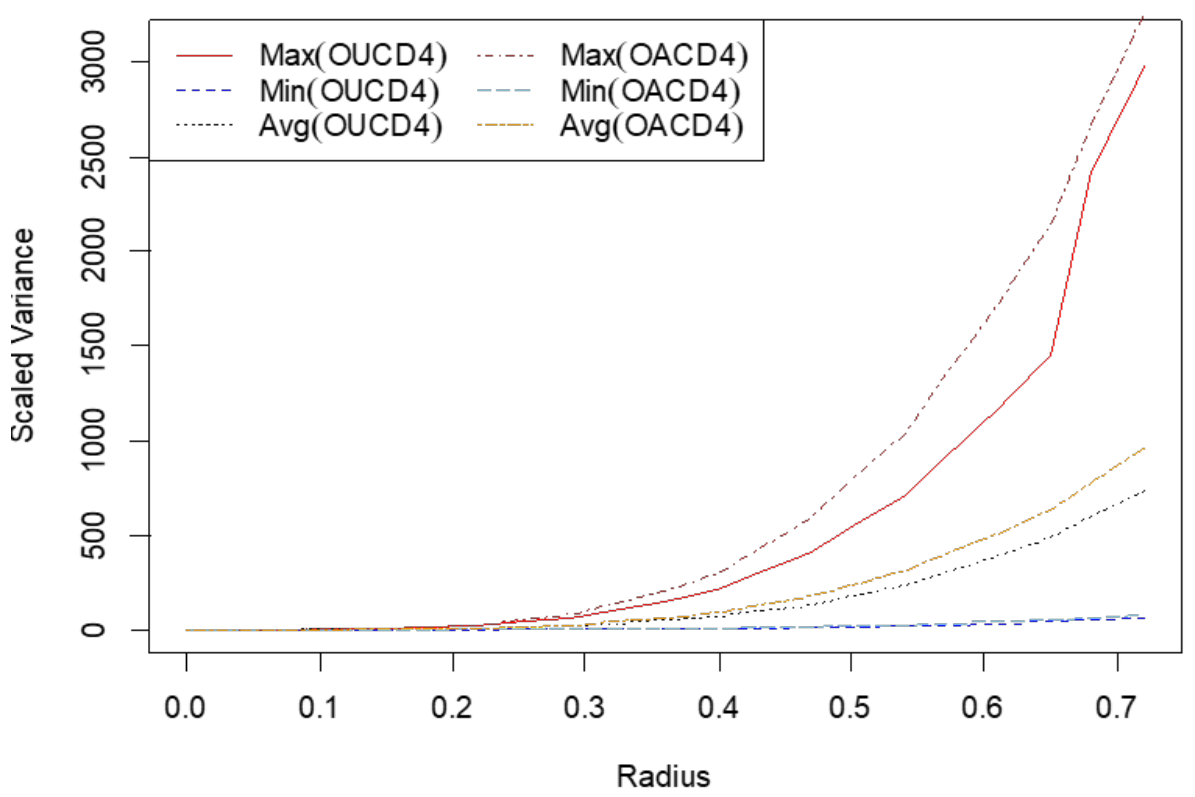

Figure 6. VDG for $\mathrm{OUCD}_{4}$ and $\mathrm{OACD}_{4}$ when $k$ is 7

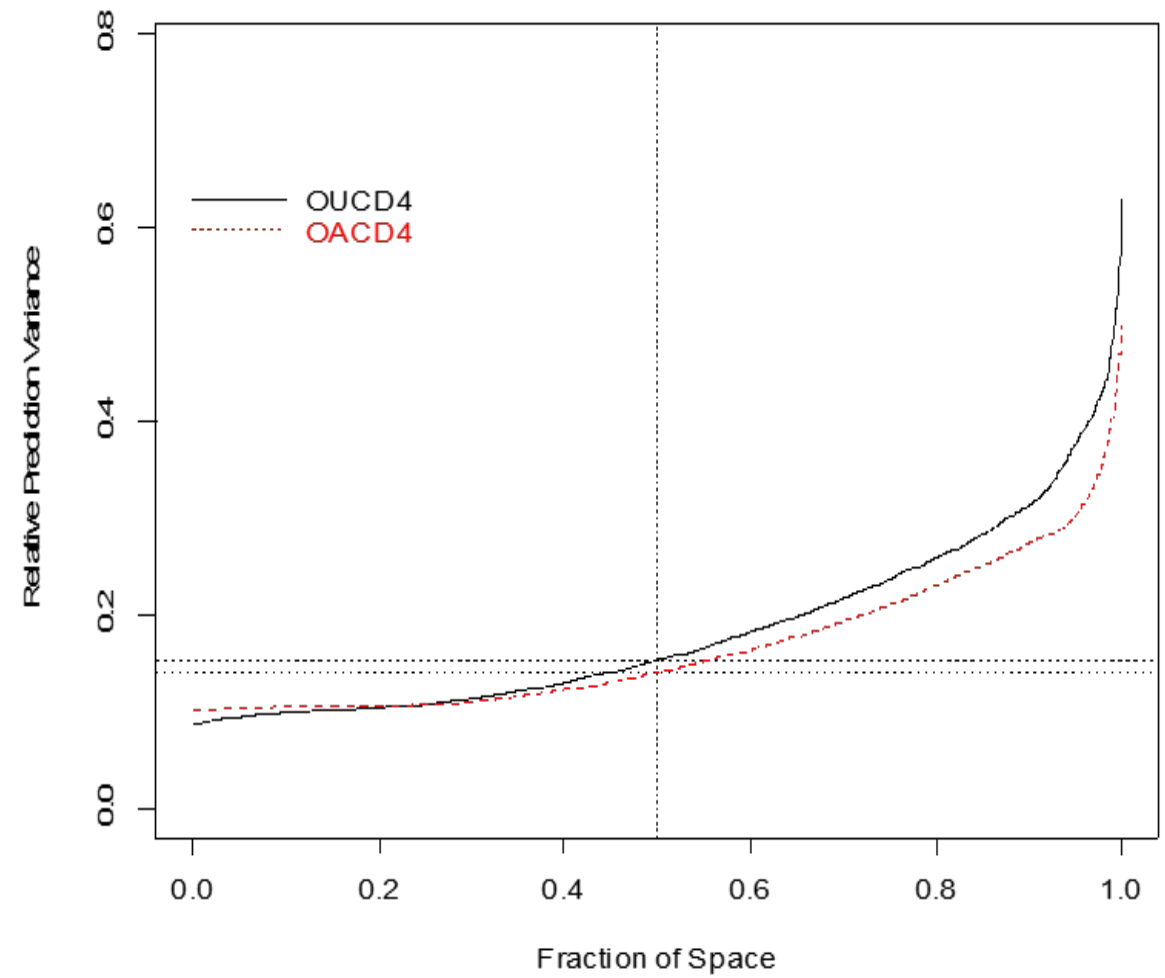


Figure 7. FDS for $\mathrm{OUCD}_{4}$ and $\mathrm{OACD}_{4}$ when $k$ is 2

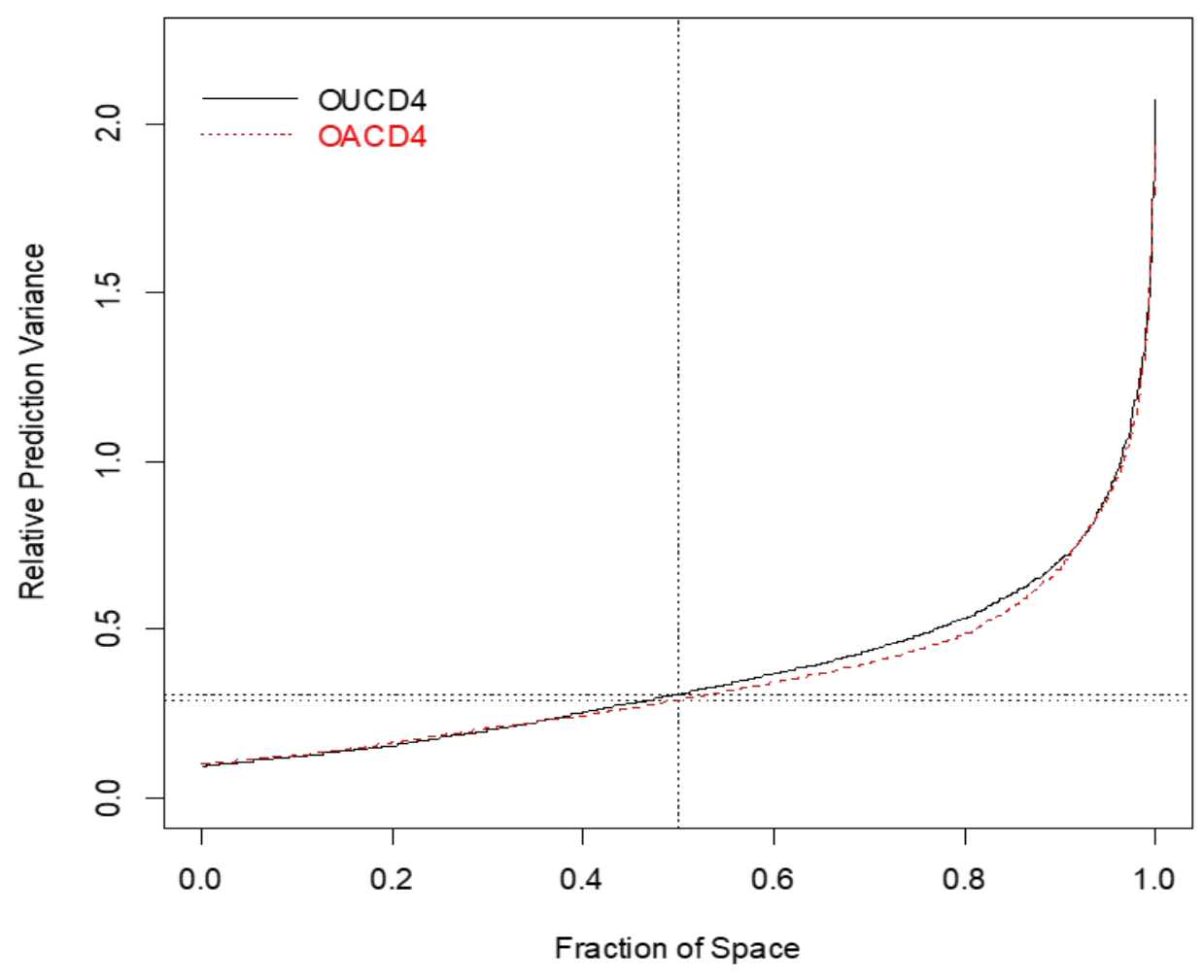

Figure 8. FDS for $\mathrm{OUCD}_{4}$ and $\mathrm{OACD}_{4}$ when $k$ is 3

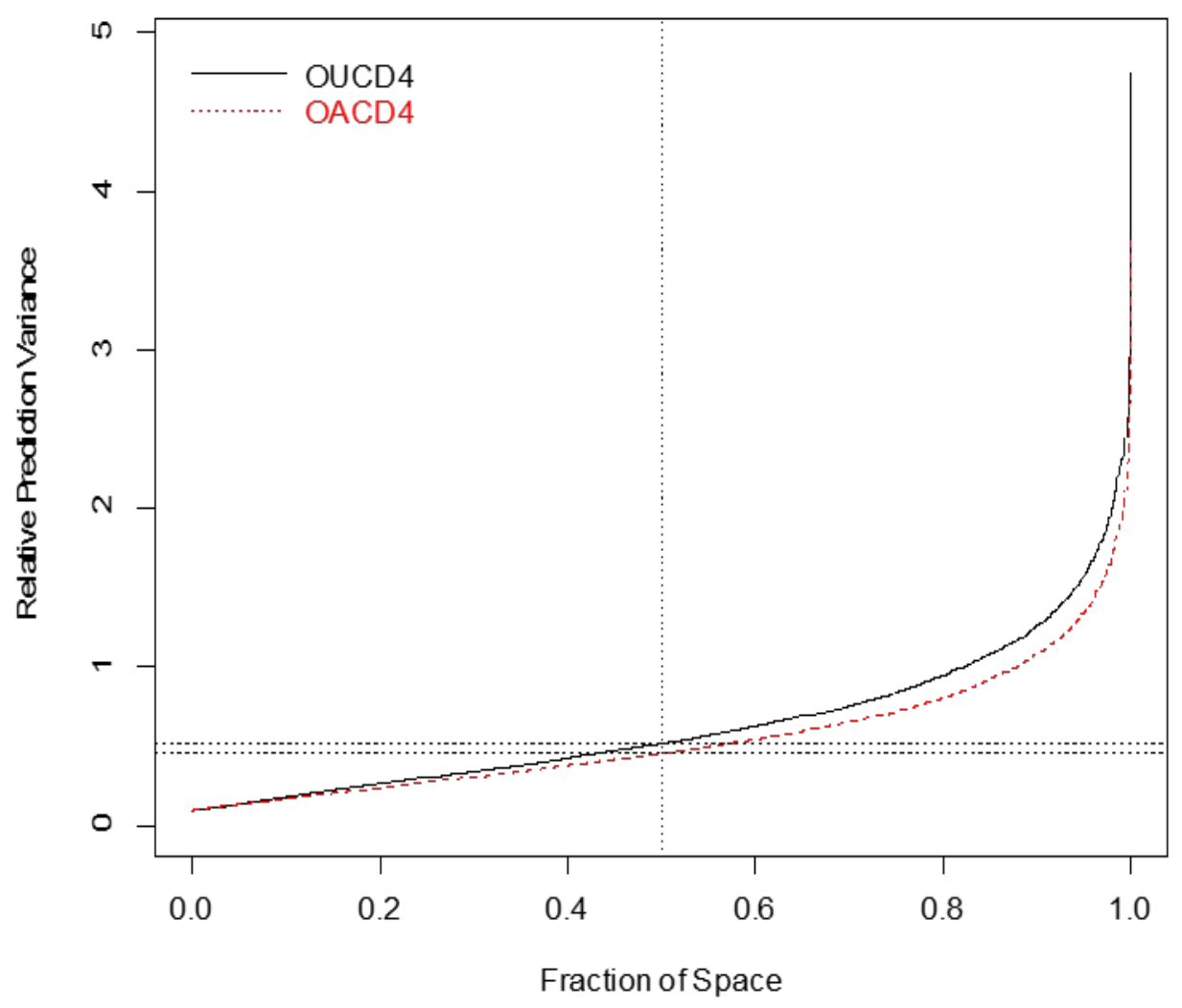

Figure 9. FDS for $\mathrm{OUCD}_{4}$ and $\mathrm{OACD}_{4}$ when $k$ is 4 


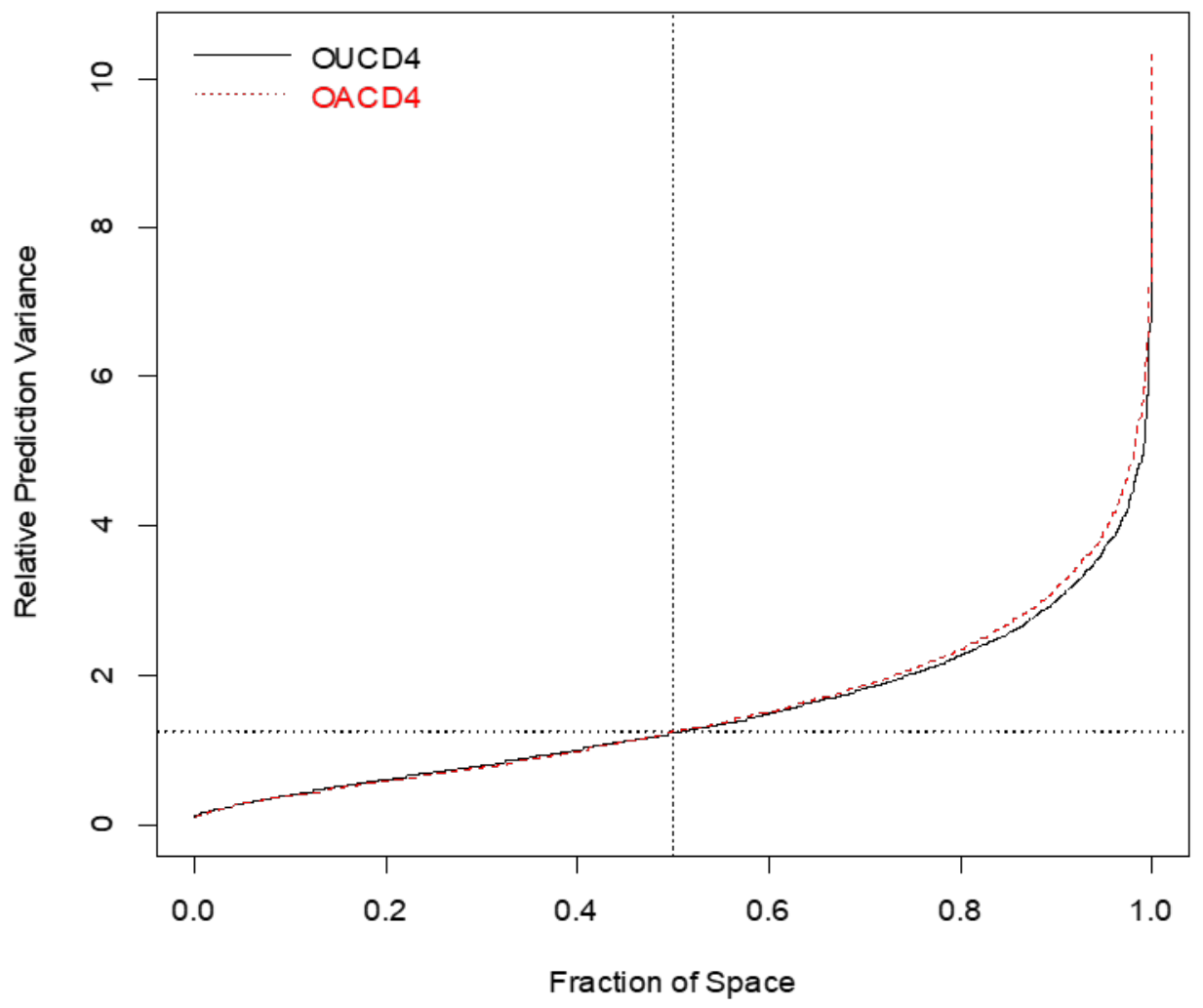

Figure 10. FDS for $\mathrm{OUCD}_{4}$ and $\mathrm{OACD}_{4}$ when $k$ is 5

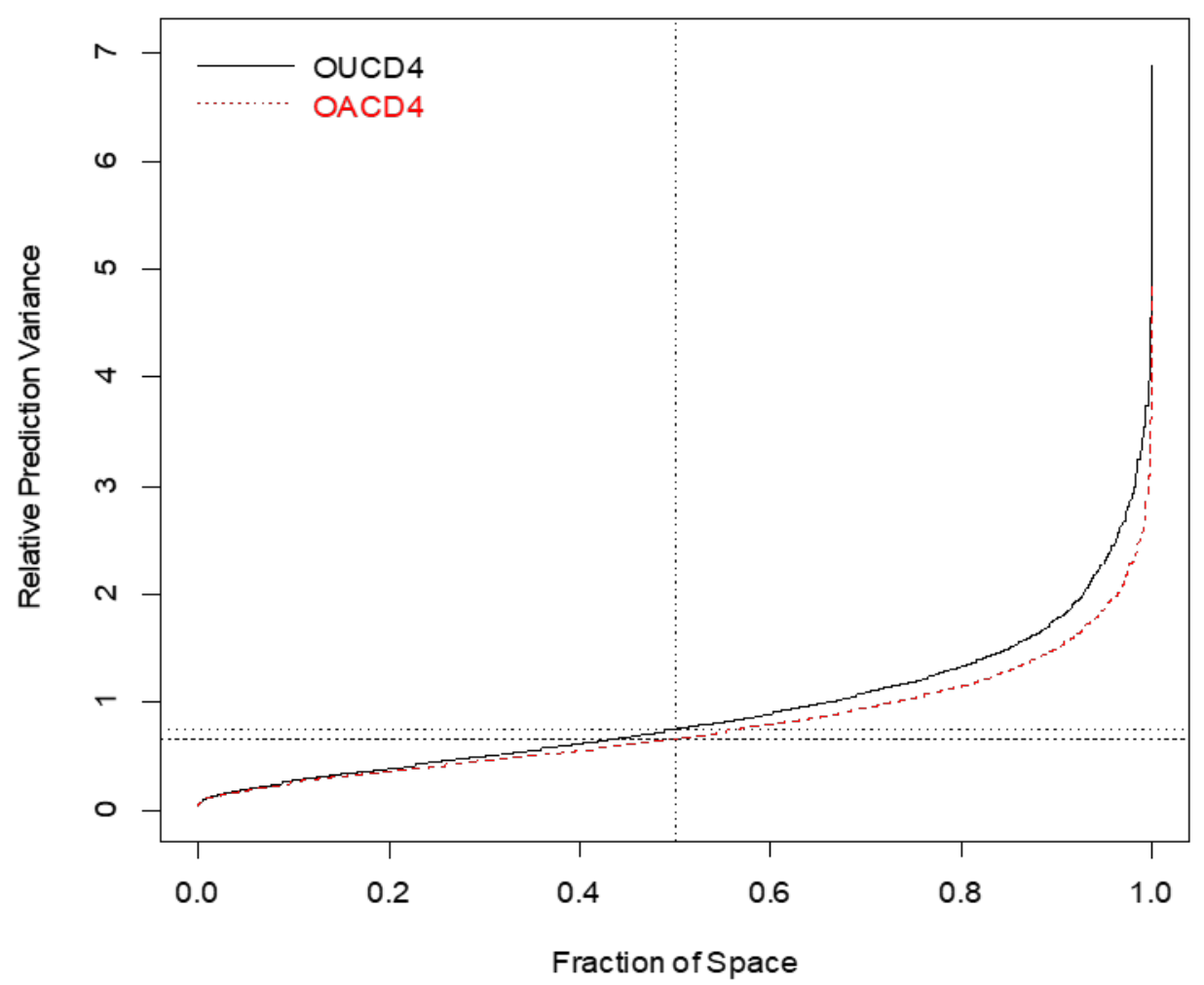

Figure 11. FDS for $\mathrm{OUCD}_{4}$ and $\mathrm{OACD}_{4}$ when $k$ is 6 


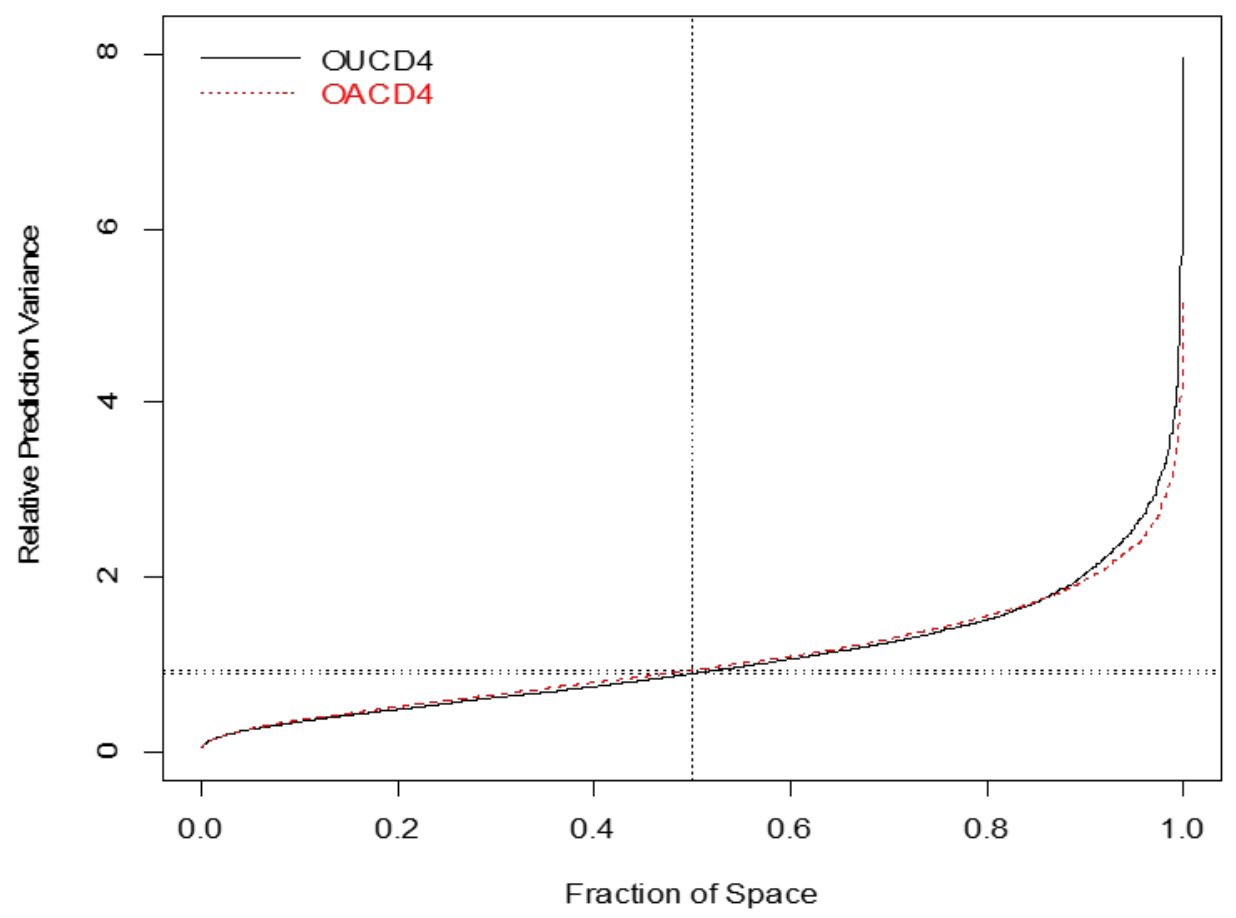

Figure 12. $\mathrm{FDS}$ for $\mathrm{OUCD}_{4}$ and $\mathrm{OACD}_{4}$ when $k$ is 7

\section{Conclusions}

This paper plotted the VDGs and FDS graphs of a new third-order response surface design called orthogonal uniform composite design $\left(\mathrm{OUCD}_{4}\right)$ and compared it with another third-order response surface design called orthogonal array composite design $\left(\mathrm{OACD}_{4}\right)$. These designs were examined in terms of singled-value optimality criteria such $D-, A$ - and $G$. It was shown that the $\mathrm{OUCD}_{4}$ performed better in terms of the singled-value criteria such as the $D-, A$ - and $G$-optimality criteria compared to the $\mathrm{OACD}_{4}$. It was also observed that the $\mathrm{OUCD}_{4}$ and $\mathrm{OACD}_{4}$ have a stable and uniform minimum and average SPV throughout the design region but the $\mathrm{OUCD}_{4}$ had an even more stable minimum and average $\mathrm{SPV}$ than $\mathrm{OACD}_{4}$ although the maximum SPV of $\mathrm{OUCD}_{4}$ was not as stable as those of $\mathrm{OACD}_{4}$ shown by the VDGs. In general, the two third-order response surface designs were performed relatively well in the spherical region. The $\mathrm{OUCD}_{4}$ was shown to have a better SPV over a fraction of design space than the $\mathrm{OACD}_{4}$ for factors 2, 3, 4 and 6, implying that the $\mathrm{OUCD}_{4}$ prediction variances were more stable and uniform over a fraction of design space than the $\mathrm{OACD}_{4}$. The VDG and FDS graphs show a clearer picture of the SPV throughout the design region and over a fraction of design space than the single-value criteria.

\section{Acknowledgement}

This work was supported by the German Academic
Exchange Service (DAAD), Bonn, Germany (Funding number: 57423580 ()-0002).

\section{REFERENCES}

[1] Draper N. R., "Third order rotatable designs in three factors: analysis". Technometrics, vol. 4, no. 2, pp. 219-234, 1962. DOI: $10.1080 / 00401706.1962 .10490006$.

[2] Rashid F., Akram M., Akbar A., Javed A., "Some new augmented fractional Box-Behnken designs", Communications in Statistics-Theory and Methods, vol. 46, no. $4, \quad$ pp. 2007-2012, 2017.DOI: $10.1080 / 03610926.2015 .1032423$.

[3] Arshad H. M., Akhtar M., Gilmour S. G., "Augmented Box-Behnken designs for fitting third-order response surfaces", Communications in Statistics-Theory and Methods, vol. 41, no. 23, pp. 4225-4239, 2012. DOI: 10.1080/03610926.2011.568154.

[4] Zhang X. R., Qi Z. F., Zhou Y. D., Yang F., "Orthogonal-array composite design for the third-order models", Communications in Statistics-Theory and Methods, vol. 47, no. 14, pp. 3488-3507, 2017. DOI: 10.1080/03610926.2017.1359297.

[5] Yang Y., "Multiple criteria third-order response surface design and comparison", Master's Thesis, Florida State University, Famu-Fsu College of Engineering, 2008. https://fsu.digital.flvc.org/islandora/object/fsu\%3A175634.

[6] Mee R. W., "Optimal three-level designs for response surfaces in spherical experimental regions", Journal of 
Quality Technology, vol. 39, no. 4, pp. 340-354, 2007. DOI: 10.1080/00224065.2007.11917700.

[7] Dutka M., Ditaranto M., Løvås T., “Application of a central composite design for the study of NOx emission performance of a low NOx burner", Energies, vol. 8, no. 5, pp. 3606-3627, 2015. DOI: 10.3390/en8053606.

[8] Kwon S. S., Park S. H., "Conditions of slope-rotatability for third-order polynomial regression models", Communications in Statistics-Theory and Methods, vol. 41, no. 23, pp. 4348-4359, 2012. DOI: 10.1080/03610926.2011.573158.

[9] Yankam B. M., Oladugba A. V., "Prediction Variance Capabilities of Third-Order Response Surface Designs for Cuboidal Regions", Mathematics and Statistics, vol. 9, no. 5, pp. 760-772, 2021. DOI: 10.13189/ms.2021.090516.

[10] Myers R. H., Vining G. G., Giovannitti-Jensen A., Myers S. L., "Variance dispersion properties of second-order response surface designs", Journal of Quality Technology, vol. 24, no. 1, pp. 1-11, 1992. DOI: 10.1080/00224065.1992.11979368.

[11] Li J., Li L., Borror C. M., Anderson-Cook C., Montgomery D. C., "Graphical summaries to compare prediction variance performance for variations of the central composite design for 6 to 10 factors", Quality Technology \& Quantitative Management, vol. 6, no. 4, pp. 433-449, 2009. DOI: 10.1080/16843703.2009.11673209.

[12] Borkowski J. J., "Spherical prediction-variance properties of central composite and Box-Behnken designs", Technometrics, vol. 37 , no. 4, pp. 399-410, 1995. DOI: 10.1080/00401706.1995.10484373.

[13] Oyejola B. A., Nwanya J. C., "Selecting the right central composite design", International Journal of Statistics and Applications, vol. 5, no. 1, pp. 21-30, 2015. DOI: 10.5923/j.statistics.20150501.04.

[14] Nwanya J. C., Dozie K. C., "Optimal Prediction Variance Capabilities of Inscribed Central Composite Designs", Asian Journal of Probability and Statistics, vol. 8, no. 1, pp. 1-8, 2020. DOI: 10.9734/AJPAS/2020/v8i130194.

[15] Borkowski J. J., "Minimum, maximum, and average spherical prediction variances for central composite and Box-Behnken designs", Communications in Statistics Theory and Methods, vol. 24, no. 10, pp. 2581-2600, 1995. DOI: $10.1080 / 03610929508831634$.
[16] Giovannitti-Jensen A., Myers R. H., "Graphical assessment of the prediction capability of response surface designs", Technometrics, vol. 31 , no. 2, pp. 159-171, 1989. DOI: 10.1080/00401706.1989.10488510.

[17] Zahran A., Anderson-Cook C. M., Myers R. H., "Fraction of design space to assess prediction capability of response surface designs", Journal of Quality Technology, vol. 35, no. 4, pp. 377-386, 2003. DOI: 10.1080/00224065.2003.11980235.

[18] Schoonees P., Le Roux N., Coetzer R., "Flexible graphical assessment of experimental designs in R: The vdg package", Journal of Statistical Software, vol. 74, no. 3, pp. 1-22, 2016. DOI: $10.18637 /$ jss.v074.i03.

[19] Borkowski J. J., "A comparison of prediction variance criteria for response surface designs", Journal of Quality Technology, vol. 35, no. 1, pp. 70-77, 2003. DOI: 10.1080/00224065.2003.11980192.

[20] Trinca L. A., Gilmour S. G., "Variance dispersion graphs for comparing blocked response surface designs", Journal of Quality Technology, vol. 30, no. 4, pp. 314-327, 1998. DOI: 10.1080/00224065.1998.11979868.

[21] Vining G. G., Myers R. H., "A graphical approach for evaluating response surface designs in terms of the mean squared error of prediction", Technometrics, vol. 33, no. 3, pp. 315-326, 1991. https://www.jstor.org/stable/1268783

[22] Wesley W. R., Simpson J. R., Parker P. A., Pignatiello Jr J. J. "Maximum and Minimum Prediction Variance for Spherical and Cuboidal Split Plot Designs", Communications in Statistics-Theory and Methods, vol. 38, no. 13, pp. 2251-2266, 2009. DOI: 10.1080/03610920802521232

[23] Chigbu P. E., Ukaegbu E. C., Nwanya J. C., "On comparing the prediction variances of some central composite designs in spherical regions: a review", Statistica, vol. 69, no. 4, pp. 285-298, 2009. DOI: https://doi.org/10.6092/issn.1973-220 $1 / 3560$.

[24] Onwuamaeze C. U. "Optimal prediction variance properties of some central composite designs in the hypercube", Communications in Statistics-Theory and Methods, vol. 50, no. 8, pp. 1911-1924, 2019. https://doi.org/10.1080/036109 26.2019 .1656746 .

[25] Zhang A., Li H., Quan S., Yang Z., "UniDOE: Uniform Design of Experiments. R package version 1.0.2", vol. 1, no. 2, pp. 1-12, 2018 